\title{
Infrared properties of a prototype model for beyond-Standard Model physics ${ }^{\dagger}$
}

\author{
Anna Hasenfratz \\ Department of Physics, University of Colorado, Boulder, CO, USA \\ E-mail: Anna.Hasenfratz@colorado.edu \\ Claudio Rebbi \\ Department of Physics, Boston University, Boston, MA, USA \\ E-mail: rebbi@bu . edu

\section{Oliver Witzel} \\ Higgs Centre for Theoretical Physics, School of Physics \& Astronomy, \\ The University of Edinburgh, Edinburgh, United Kingdom \\ E-mail: o.witzelded.ac.uk
}

\begin{abstract}
We construct a prototype BSM model based on the SU(3) color gauge group and a combination of 4 light (massless) and 8 heavy flavors. In the infrared, the SU(4) flavor chiral symmetry is spontaneously broken, while in the ultraviolet this model exhibits the properties of the $N_{f}=$ 12 conformal fixed point. Renormalization group considerations predict the spectrum of such a system to show hyperscaling, i.e. dimensionless ratios of hadron masses or decay constants are independent of the heavy mass. Hyperscaling is present for bound states of light, heavy, or a combination of heavy and light flavors and leads to a strongly predictive model. Despite chiral symmetry breaking, this system features a spectrum exhibiting a very non-QCD like behavior. Furthermore, the gauge coupling becomes an irrelevant parameter. We support these expectations by presenting numerical results based on four different values of the heavy quark mass $a m_{h}$, up to six different values of the light quark mass $a m_{\ell}$, and include, for the first time, preliminary data at a second value of the gauge coupling $\beta$. Our model can be embedded in scenarios describing the Higgs boson either as a pseudo Nambu-Goldstone boson or a dilaton-like particle.
\end{abstract}

34th annual International Symposium on Lattice Field Theory

24-30 July 2016

University of Southampton, $U K$

\footnotetext{
$\dagger$ This article combines the contribution "Infrared properties of a prototype pNGB model for BSM physics" by Anna Hasenfratz and the contribution "Spectrum of a prototype model with the Higgs as pNGB" by Claudio Rebbi.

${ }^{\ddagger}$ Speaker.
} 


\section{Introduction}

While the analysis of data accumulated by ATLAS and CMS in 2016 do not provide compelling evidence for beyond-Standard Model (BSM) phenomena [1,2], it is generally accepted that the Standard Model (SM) is only an effective theory. New interactions are needed for an ultraviolet (UV) completion of the Higgs sector, which otherwise leads to a triviality in the UV, as well as to explain other phenomena, such as dark matter, for which there is tantalizing evidence but no established theory. Many of the models which are currently considered for BSM physics are based on a large scale separation between the infrared (IR) and ultraviolet (UV) regimes [3-8], that leads to a "walking" gauge coupling and provides a dynamical mechanism for electroweak (EW) symmetry breaking. This mechanism avoids tuning of the Higgs mass within a framework which may satisfy EW precision measurement constraints. Figure 1 illustrates the assumed transition between the UV and IR regimes. An almost universal features of all strongly coupled BSM models is that they

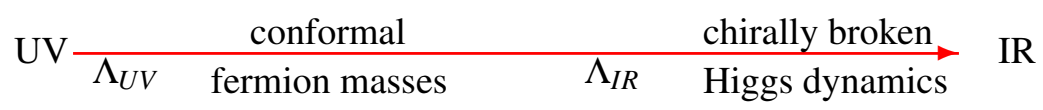

Figure 1: Schematic representation of the transition between UV and IR regimes in strong dynamics models for EW symmetry breaking.

assume the proximity of an IR fixed point, which is eventually responsible for the walking of the gauge coupling. This in turn requires the presence of a sufficient number of fermions to push the theory close to the conformal window, whose presence is indicated by perturbative $[9,10]$ as well as numerical arguments (for recent references see [11, 12]). In order to investigate the effect which a large number fermions will have on the transition between the UV and IR regimes, in collaboration with Richard Brower and Evan Weinberg, we embarked in the numerical study of an SU(3) theory with four light fermions and eight heavy fermions of variable mass [6, 13-17] The original goal was to see how, through the variable mass, one could interpolate from the confining behavior of a QCD-like theory to the conformal behavior of a theory with 12 flavors of (almost) massless fermions. The investigation led to some interesting, unique results: While we did observe a changing behavior of the renormalized coupling, which exhibited a growing region of slow walking as the mass of the eight heavy fermions decreased, the spectrum of light composites with a properly rescaled lattice spacing did not appear to change with the heavy fermions mass. A result which we interpreted as evidence of hyperscaling, a consequence of infrared conformal behavior and in agreement with step scaling studies of the 12-flavor system [18]. In these proceedings we report on an extension of that early work, where we examine in detail the spectrum of heavy composites, finding results which further confirm hyperscaling and may have implications for future observations at the LHC. We also performed simulations at a different value of the gauge coupling constant to validate the notion that the gauge coupling is an irrelevant parameter in the neighborhood of an IR fixed point.

\section{Hyperscaling}

We start from the premise that in the limit where all twelve flavors are massless our theory has an infrared fixed point (IRFP). This assumption has substantial numerical support [19-23] and 
remaining concerns $[24,25]$ were recently addressed in Ref. [18]. In the basin of attraction of the IRFP a Wilsonian renormalization group analysis can be used to investigate how changes in the bare fermion masses effect physical observables. Working with the lattice regularization and inside the conformal window, the bare parameters of the theory can be separated into irrelevant gauge couplings $g_{i}$ and relevant lattice masses $\widehat{m}_{i}=a m_{i}$. The critical surface is given by $\widehat{m}_{i}=0$ where the system is conformal at the IRFP $g_{i}^{\star}$.

In the neighborhood of the IRFP an RG transformation that changes the scale $\mu \rightarrow \mu^{\prime}=$ $\mu / b(b>1)$ drives the gauge couplings to $g_{i}^{\star}$, while masses transform with the scaling dimension $y_{m}=1+\gamma_{m}$ as $\widehat{m}_{i} \rightarrow \widehat{m}_{i}^{\prime}=b^{y_{m}} \widehat{m}_{i}$. The correlation function of an operator $H$, after rescaling all dimensional quantities by $b$, change as

$$
C_{H}\left(t ; g_{i}, \widehat{m}_{i}, \mu\right)=b^{-2 y_{H}} C_{H}\left(t / b ; g_{i}^{\prime}, \widehat{m}_{i}^{\prime}, \mu\right),
$$

where $y_{H}$ is the scaling dimension of $H[26,27]$.

As $b$ increases the fermion mass increases and eventually the fermions decouple from the IR dynamics when the mass reaches the cutoff at $\widehat{m}_{i}^{\prime} \sim \mathscr{O}(1)$.

This analysis can be extended to the case when there are two different fermions masses $\widehat{m}_{h}=$ $a m_{h}$ and $\widehat{m}_{\ell}=a m_{\ell}$, as in our model, with $\widehat{m}_{h} \geq \widehat{m}_{\ell}$. Since both masses scale with the same exponent $y_{m}$, the dependence on $\widehat{m}_{i}^{\prime}=\left(\widehat{m}_{h}^{\prime}, \widehat{m}_{\ell}^{\prime}\right)$ in Eq. (2.1) can be replaced with $\left(\widehat{m}_{h}^{\prime}, \widehat{m}_{\ell} / \widehat{m}_{h}\right)=\left(\widehat{m}_{h}^{\prime}, m_{\ell} / m_{h}\right)$

$$
C_{H}\left(t ; g_{i}, \widehat{m}_{i}, \mu\right)=b^{-2 y_{H}} C_{H}\left(t / b ; g_{i}^{\prime}, \widehat{m}_{h}^{\prime}, m_{\ell} / m_{h}, \mu\right) .
$$

The heavy fermions decouple when $\widehat{m}_{h}^{\prime}=b^{y_{m}} \widehat{m}_{h}=\mathscr{O}(1)$. Below that scale the dependence on $\widehat{m}_{h}$ is through the ratio $m_{\ell} / m_{h}$. The infrared limit for the light flavors can be set at $b=\widehat{m}_{\ell}^{-1 / y_{m}}$. Then Eq. (2.2) reduces to

$$
C_{H}\left(t ; g_{i}, \widehat{m}_{i}, \mu\right)=\widehat{m}_{\ell}^{2 y_{H} / y_{m}} C_{H}\left(t \widehat{m}_{\ell}^{1 / y_{m}} ; g_{i}^{\prime}, m_{\ell} / m_{h}, \mu\right) .
$$

Correlation function behave exponentially at large distances,

$$
C_{H}\left(t ; g_{i}, \widehat{m}_{i}, \mu\right) \propto e^{-M_{H} t}, \quad t \rightarrow \infty .
$$

Comparison of the $t$ dependence in Eqs. (2.3) and (2.4) produces the scaling relation

$$
a M_{H}=\left(\widehat{m}_{\ell}\right)^{1 / y_{m}} F_{H}\left(m_{\ell} / m_{h}\right) .
$$

Assuming that $b$ is large enough that the gauge couplings take their IRFP value, $g_{i}^{\prime}=g_{i}^{\star}, F_{H}$ will be a function of $m_{\ell} / m_{h}$ only, independent of the bare gauge couplings. Ratios of masses

$$
\frac{M_{H 1}}{M_{H 2}}=\frac{F_{H 1}\left(m_{\ell} / m_{h}\right)}{F_{H 2}\left(m_{\ell} / m_{h}\right)}
$$

depend only on $m_{\ell} / m_{h}$, while the scaling function $F_{H 1} / F_{H 2}$ can of course be different for different observables.

As we will see in the next section, our results for light fermion composites and of heavy fermion composites, corroborate this prediction: composite masses and decay constants obtained with different $m_{\ell}$ and $m_{h}$ fall on universal curves as function of $m_{\ell} / m_{h}$. We expect the same to hold 


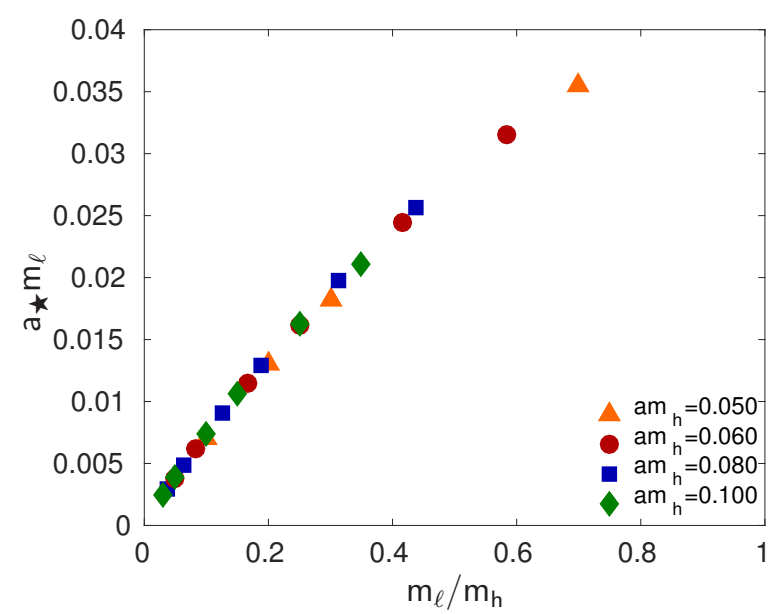

Figure 2: The masses $a_{\star} m_{\ell}$ displayed as function of the ratios $m_{\ell} / m_{h}$. Universality implies that they should fall on a single curve.

for the spectrum of heavy-light composites, but did not verify it by numerical simulations. Small deviations from universality can arise from corrections to scaling due to the slowly running gauge coupling, i.e. deviations from $g_{i}^{\prime}=g_{i}^{\star}$. We have investigated these corrections within the $N_{f}=12$ system and a similar analysis could be repeated here [22]. We have not done this, though, in our present work.

\section{Numerical results}

We simulated an SU(3) system with one staggered field (= 4 flavors) with light mass $m_{\ell}$ and two staggered fields ( $=8$ flavors) with heavy mass $m_{h}$. Simulations were done with $a m_{\ell}=$ $0.003,0.005,0.010,0.015,0.025,0.035$, and $a m_{h}=0.050,0.060,0.080,0.100$. We used fundamental-adjoint gauge action with $\beta=4.0, \beta_{a}=-\beta / 4[22,28]$ and nHYP-smeared staggered fermions [29]. Recently we also performed simulations with $\beta=4.4$. Lattice sizes were mostly $24^{3} \times 48$ and $32^{3} \times 64$, but also $16^{3} \times 32$ (exploratory), $36^{3} \times 64$ and $48^{3} \times 96$. The lattice generation was done with the hybrid Monte Carlo algorithm with one Hasenbusch intermediate mass; most simulations/measurements were performed with FUEL [30, 31]; most calculations were done with USQCD SciDAC software on USQCD computers at Fermilab and NSF-MRI computers at the MGHPCC. Disconnected diagrams (for the $0^{++}$singlet states) were computed with stochastic sources (6 sources, full color and time dilution, even-odd space dilution.) We used the gradient flow method to set the scale for the various lattices. The gradient flow defines the renormalized coupling $g_{\mathrm{GF}}$ by $g_{G F}^{2}(\mu=1 / \sqrt{8 t})=t^{2}\langle E(t)\rangle / \mathscr{N}$ where $t$ is the flow time, $E(t)$ is the action density and $\mathscr{N}$ is conventionally taken as $3\left(N_{c}-1\right) /\left(128 \pi^{2}\right)$ [32-34]. We set the scale demanding that $g_{G F}^{2}\left(t=t_{0}\right)=0.3 / \mathscr{N}$. The lattice flow time $t_{0} / a^{2}$ then defines the scale. The symbol $a_{\star}$ appearing in some of the following figures is used to provide a common scale for all dimension-full quantities. It denotes the lattice spacing for the simulation on the $36^{3} \times 64$ lattice with $a m_{\ell}=0.003, a m_{h}=0.080$. The relation between $a_{\star} m_{\ell}$ and the ratios $m_{\ell} / m_{h}$ is worth noting. As explained in the previous section, we expect dimensionless ratios of physical quantities to depend on the ratio $m_{\ell} / m_{h}$ but not individually on $m_{\ell}$ or $m_{h}$. Hyperscaling manifests itself also if 

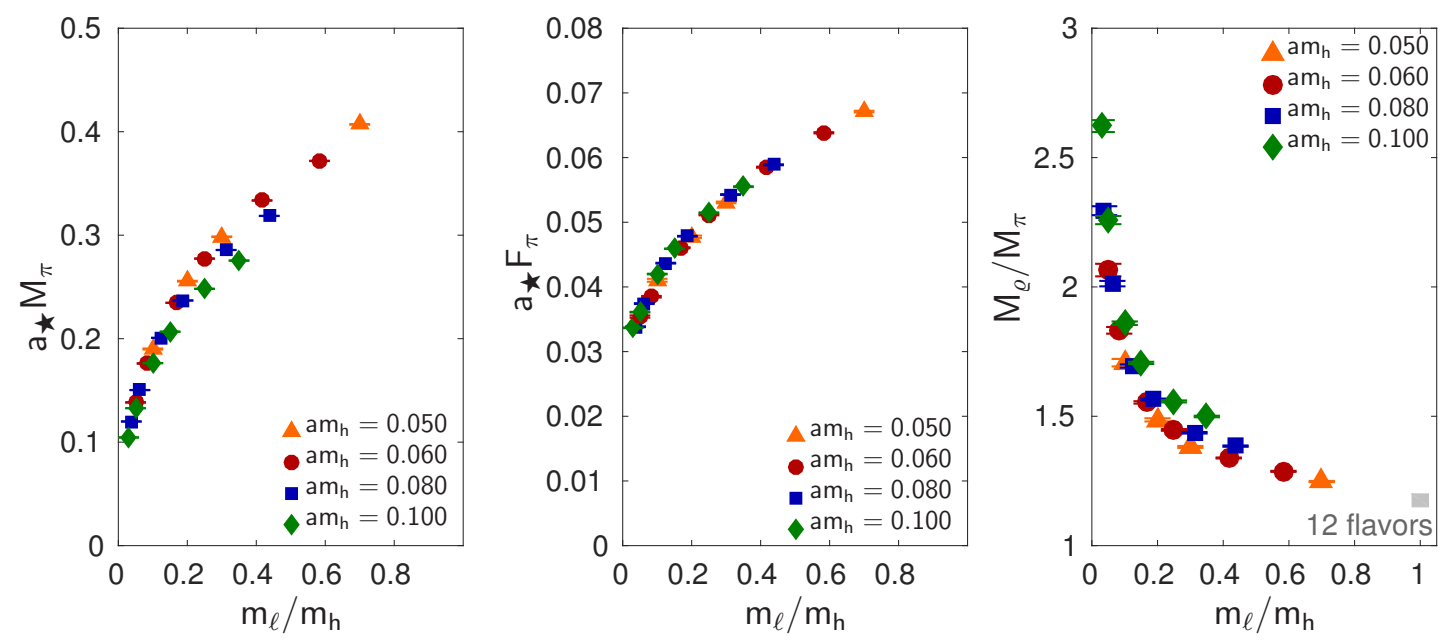

Figure 3: The left panel shows the pseudoscalar meson mass $M_{\pi}$ and the panel in the middle its decay constant, $F_{\pi}$, as functions of $m_{\ell} / m_{h}$. Note the universality as well as the fact that the pseudoscalar mass tends to zero in the chiral limit while $F_{\pi}$ tends to a finite limit, albeit with a steep drop. The right panel shows the ratio between the vector meson mass $m_{\rho}$ and $m_{\pi}$, which diverges in the chiral limit with chiral symmetry breaking, while it should tend to a constant in a conformal theory. (We use hadron spectroscopy notation to denote the states in our model and show only statistical errors.)

quantities are plotted in terms of $a_{\star} m_{\ell}$ because $a_{\star}$ can be considered as another physical quantity. ${ }^{1}$ The two variables $a_{\star} m_{\ell}$ and $m_{\ell} / m_{h}$ should therefore track each other falling on a single curve and this is apparent in Fig 2.

The first question one should address is whether the system with our parameter values exhibits chiral symmetry breaking and is not conformal. Figure 3 provides strong evidence for that: in the chiral limit $M_{\pi}$ goes to zero, $F_{\pi}$ is finite, and $M_{\rho} / M_{\pi}$ diverges.
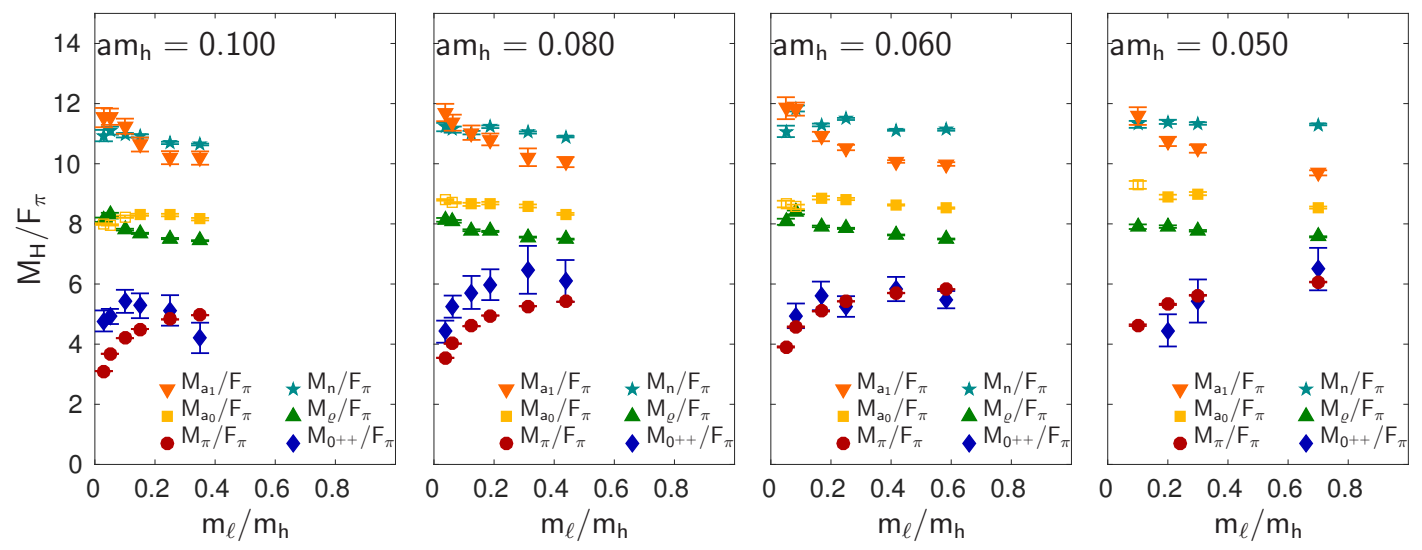

Figure 4: The pion, rho, isosinglet $0^{++}$and isomultiplet $a_{0}$ scalar, axial, and nucleon mass of the light-light flavor spectrum in units of $F_{\pi}$ (errors are statistical only). The four panels show the $N_{f}=4+8$ spectrum as the function of the ratio $m_{\ell} / m_{h}$ for $a m_{h}=0.100,0.080,0.060$, and 0.050 .

\footnotetext{
${ }^{1}$ We recall that $m_{\ell}$ stands for the fixed physical value of a light fermion mass. Thus the values of $a_{\star} m_{\ell}$ are different from the quoted values for $a m_{\ell}$. Unlike in QCD, the lattice spacing $a$ depends strongly on $m_{\ell}$ and $m_{h}$.
} 

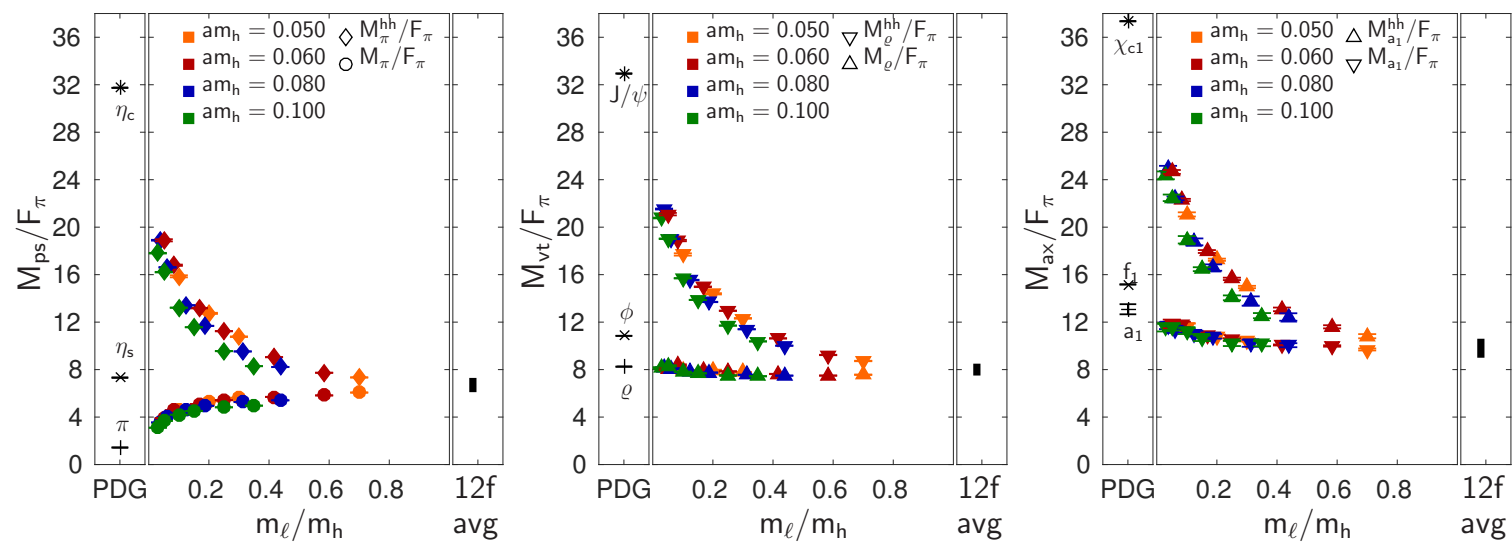

Figure 5: The three set of panels show pseudoscalar (ps), vector (vt), and axial (ax) meson masses in units of $F_{\pi}$. The wide central panels show our data (with statistical errors only) as function of $m_{\ell} / m_{h}$ and different colors indicate the different $m_{h}$ values. The small panels to the right show averaged values for degenerate 12 flavors [22, 24, 35, 36] and the panels on the left the corresponding PDG values [37] for QCD divided by $F_{\pi}=94 \mathrm{MeV}$. Values for the corresponding bottomonium states $\left(\eta_{b}, \Upsilon\right.$, and $\left.\chi_{b 1}\right)$ are too heavy to be shown on a reasonable scale. While the pseudoscalar and vector states are in general well understood in QCD, pure $(s \bar{s})$ states do not occur in nature. For the $\eta_{s}$ mass, we use the lattice determination, $M_{\eta_{s}}=688.5(2.2)$ $\mathrm{MeV}$ [38], and quote for the vector and axial the PDG entries for the $\phi(1020)$ and $f_{1}(1420)$, respectively. Regardless of ambiguities in the QCD values, these plots highlight the different character of our heavy-heavy spectrum. Due to the presence of an IRFP, the system shows hyperscaling and we observe independence of the $m_{h}$, an unusual behavior in QCD standards.

Figure 4 shows the masses of the light-light composites as dimensionless ratios over $F_{\pi}$ for the four values of the heavy fermion masses, separately, plotted as a function of the dimensionless ratio $m_{\ell} / m_{h}$. Superimposing the panels, which we do in a later figure, would show hyperscaling, but here we keep the data apart for clarity of presentation. An interesting and possibly very important feature, already noticed in [6] and by other researchers, is that the mass of the singlet scalar meson appears to track the pseudoscalar mass through the range of light fermion masses. If this were to continue down toward the chiral limit, it would indicate that BSM theories based on strong dynamics may give origin to a light scalar meson, as would be necessary for an interpretation of the Higgs as a dilaton-like composite state.

Figure 5 captures the essence of our new results. The data for the masses of light-light and heavy-heavy pseudoscalar, vector and axial vector states obtained with the four different heavy fermion masses are all plotted together as function of $m_{\ell} / m_{h}$. The data strikingly fall on universal curves as expected from hyperscaling. The small deviations can be explained by remaining lattice artifacts and scaling violations due to a slow approach to the IRFP.

The patterns of masses of the heavy-heavy composites shown in Fig. 5 bend noticeably upward as one approaches the light fermions' chiral limit. This is, however, to a large extent a consequence of the fact that, in order to display dimensionless quantities, we plot the ratios $M^{\text {hh }} / F_{\pi}$. As one can see from the central panel of Fig. $3, F_{\pi}$ exhibits a rather sharp decrease as one approaches the chiral limit and this causes an upward bend of the ratios $M^{\mathrm{hh}} / F_{\pi}$. In Figure 6 we show the masses of the heavy-heavy $\pi$ and $a_{1}$ states measured against the light-light (left panel) and heavy-heavy (right panel) $\rho$ masses. While the ratios $M^{\text {hh }} / M_{\rho}$ still exhibit an upward bend, due to the fact that, like $F_{\pi}$, 
the light-light $M_{\rho}$ also bends downward as one approaches the chiral limit (this was not explicitly shown, but can be inferred from the almost constancy of $M_{\rho} / F_{\pi}$, see Fig. 5), the ratios $M^{\text {th }} / M_{\rho}^{\text {hh }}$ show little variation with $m_{\ell} / m_{h}$. The fact remains, though, that in phenomenological applications it would be the light-light $F_{\pi}$ which sets the scale of the electroweak symmetry breaking and thus the increase of the ratios $M^{\text {hh }} / F_{\pi}$ would be of phenomenological relevance. And this brings us to an important consideration, discussed in the following paragraph.

Our system is a prototype pseudo Nambu-Goldstone boson (PNGB) model (for recent work see e.g. [3, 39-41]) in the sense that, while in no way it can be a realistic model of EWSB, it exhibits some of the main features of an eventual full fledged theory of EWSB based on strong dynamics: we expect that such a theory would have an IRFP in the limit of massless fermions in order to allow for the required separation of scales, and that it would have extra fermions, beyond the light fermions giving origin to the NG bosons, to push it toward the conformal window. Then one obvious question is how the extra fermions should be handled and what would be the implications of their existence. Would their mass represent an additional parameter in the theory? Would they imply a spectrum of composites whose masses will depend on the constituent fermion mass? and if so how should this mass be tuned? The answer that we gather from our investigation is that, because of the IRFP, the spectrum of all composite states would show hyperscaling. As a consequence the spectrum of composites would be largely independent from the bare mass of the additional fermions. Tuning it would only serve to change the range of walking. The actual spectrum of composites would of course depend on the underlying theory and it would constitute a set of excitations which would show up at some point in high energy collisions.

Our final consideration is about our choice of the value $\beta=4.0$ for the gauge coupling constant. We have argued that $\beta$ is an irrelevant parameter and thus its specific value should not matter. Changing the gauge coupling near an IRFP is similar to changing the gauge action in a QCD simulation by adding irrelevant terms. The Wilson, Symanzik, Iwasaki or fixed point actions differ only in irrelevant terms and have the same continuum limit. In the same way conformal systems with different gauge coupling values near the IRFP have the same continuum limit. Just like it is possible to choose a gauge action with reduced cutoff corrections in QCD, it is possible to choose a $\beta$ value with reduced corrections to conformal hyperscaling. Both the finite size scaling analysis of Ref. [22] and the step scaling function study of Ref. [18] indicate that $\beta \approx 4.5$ is optimal, showing minimal corrections to scaling in the $N_{f}=12$ system with our choice of gauge action. At the same time larger $\beta$ values correspond to a smaller lattice spacing for the same bare mass, requiring simulations on larger volumes. We chose $\beta=4.0$ as a compromise after substantial preliminary numerical exploration.

Nonetheless we decided that it would be good to verify the expectation that $\beta$ is an irrelevant parameter by running a simulation at a different value of the gauge coupling constant. Thus we recently started simulations increasing $\beta$ by $10 \%$. Using $32^{3} \times 64$ volumes, $\beta=4.4$, and $a m_{h}=0.070$ we are generating four ensembles with $a m_{\ell}=0.009,0.013125,0.0175$, and 0.0245 . Carrying out spectrum measurements on the currently available gauge field configurations, we show our preliminary results for pseudoscalar, vector, and axial states by the purple symbols in Fig. 7 and observe that the purple symbols ( $\beta=4.4$ data) follow closely the lines traced by the $\beta=4.0$ data. This supports the notion that the gauge coupling constant is an irrelevant parameter in the neighborhood of the IRFP. In the future, we plan to investigate finite volume effects in greater detail and will 

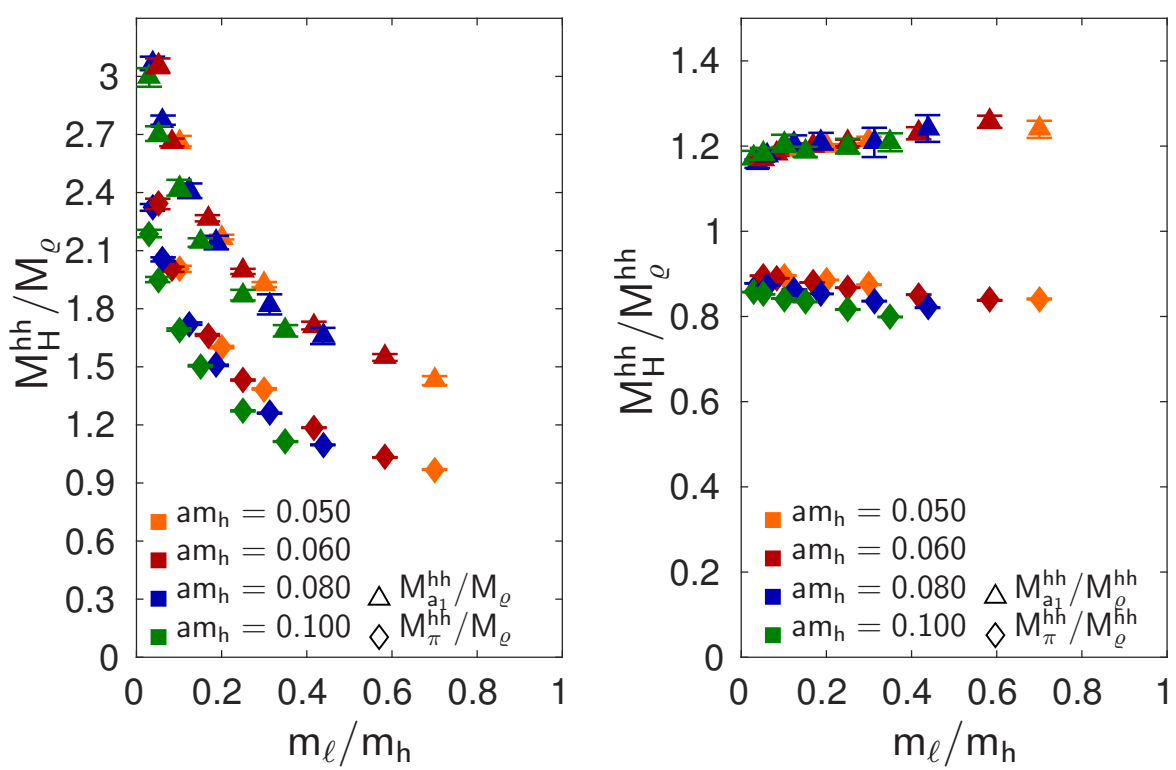

Figure 6: The masses of the heavy-heavy $\pi$ and $a_{1}$ states (with statistical errors only) measured against the light-light (left panel) and heavy-heavy (right panel) $\rho$ states.

also study scaling violations more carefully by directly comparing data at different $\beta$-values but the same $m_{\ell} / m_{h}$ ratio.

\section{Conclusions}

We simulated an SU(3) gauge system with four light and eight heavy flavors of variable mass to study the dependence of the spectrum of composite excitations on the mass of the heavy flavors. We found strong evidence of hyperscaling, with the masses of the light-light and heavy-heavy composites falling on universal lines independently of the heavy fermion mass. We emphasize this finding is solely based on analyzing dimenionless ratios and hence independent of how a lattice scale is defined. Although this was not the primary purpose of our investigation, our results lend credence to the notion that the SU(3) theory with 12 massless flavors has an infrared fixed point.

Our results show that in our setup the heavy fermion mass plays a role similar to the role of the gauge coupling constant $\beta$ in QCD simulations. In QCD $\beta$ is a (marginally) relevant parameter and the QCD spectrum is largely independent of $\beta$ (apart of course from discretization errors). The only effect of a change of $\beta$ is a corresponding change of scale. Similarly, in our system a change of $m_{h}$ has no effect on the spectrum of excitations (apart from higher order corrections) and only modifies the length of the walking window; the gauge coupling $\beta$ is now an irrelevant parameter. Since our model is based on a conformal fixed point where both the light and heavy fermion masses scale with the same anomalous dimension, physical quantities show hyperscaling and depend only on the ratio of fermion masses. This is very restrictive and qualitatively different from QCD. Thus our model, and in general models based on a conformal fixed point, are an interesting alternative to describe chirally broken systems with unique properties.

Finally, our investigation indicates that realistic strongly coupled BSM models, if they incorporate heavier mass fermions and have a dynamics governed by an IRFP, would exhibit a spectrum 


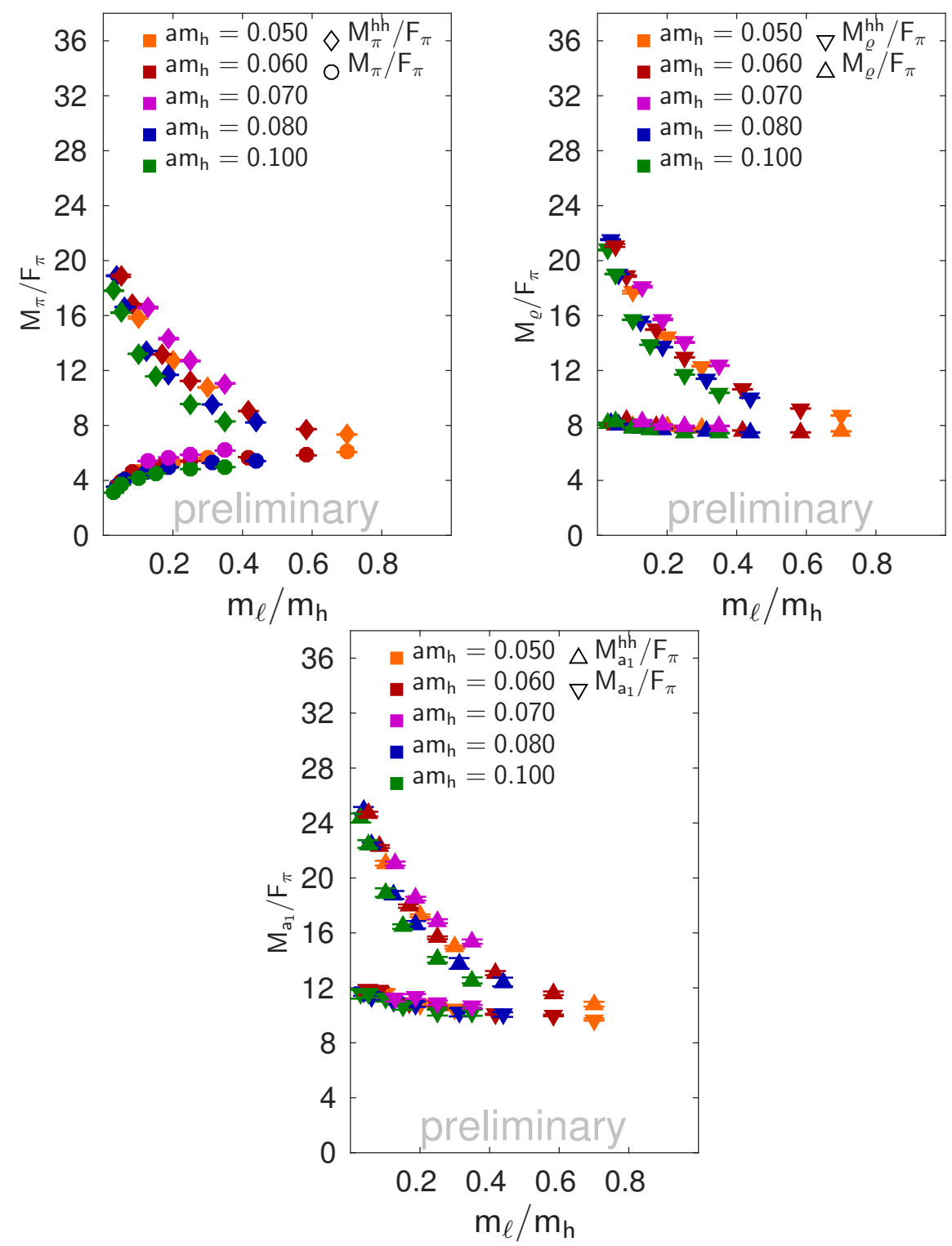

Figure 7: The same masses in units of $F_{\pi}$ as shown in Fig. 5 but supplemented here with preliminary data obtained with a different value of the bare gauge coupling constant (the purple points, with $\beta=4.4$ and $a m_{h}=0.70$ ); all errors are statistical only. The fact that the new data fall on the same curves as in Fig. 5 confirms that the bare gauge coupling is an irrelevant parameter in the neighborhood of the IRFP.

of heavy-heavy excitations of great phenomenological interest. Even more interesting are heavylight states because they would couple directly to SM particles through the light fermions. While we have not investigated the heavy-light spectrum, those states are expected to lie between the light-light and heavy-heavy spectrum, making them accessible to experiments if BSM physics is described by a model similar to ours. 


\section{Acknowledgments}

The authors thank their colleagues in the LSD Collaboration for fruitful and inspiring discussions. Computations for this work were carried out in part on facilities of the USQCD Collaboration, which are funded by the Office of Science of the U.S. Department of Energy, on computers at the MGHPCC, in part funded by the National Science Foundation, and on computers allocated under the NSF Xsede program to the project TG-PHY120002. We thank Boston University, Fermilab, the NSF and the U.S. DOE for providing the facilities essential for the completion of this work. A.H. acknowledges support by DOE grant DE-SC0010005 and C.R. by DOE grant DESC0015845. This project has received funding from the European Union's Horizon 2020 research and innovation programme under the Marie Skłodowska-Curie grant agreement No 659322.

\section{References}

[1] D. Charlton, "Highlights from the LHC (I)," (2016), plenary talk at ICHEP 2016, Chicago

[2] T. Camporesi, "LHC results Highlights," (2016), plenary talk at ICHEP 2016, Chicago

[3] R. Contino, Proceedings TASI 09, 235 (2011), arXiv:1005.4269 [hep-ph]

[4] M. A. Luty and T. Okui, JHEP 09, 070 (2006), arXiv:hep-ph/0409274 [hep-ph]

[5] D. D. Dietrich and F. Sannino, Phys. Rev. D75, 085018 (2007), arXiv:hep-ph/0611341 [hep-ph]

[6] R. C. Brower, A. Hasenfratz, C. Rebbi, E. Weinberg, and O. Witzel, Phys. Rev. D93, 075028 (2016), arXiv:1512.02576 [hep-ph]

[7] C. Csaki, C. Grojean, and J. Terning, Rev. Mod. Phys. 88, 045001 (2016), arXiv:1512.00468 [hep-ph]

[8] N. Arkani-Hamed, R. T. D'Agnolo, M. Low, and D. Pinner, JHEP 11, 082 (2016), arXiv:1608.01675 [hep-ph]

[9] T. A. Ryttov and R. Shrock, Phys. Rev. D83, 056011 (2011), arXiv:1011.4542

[10] T. A. Ryttov and R. Shrock, (2016), arXiv:1610.00387

[11] T. DeGrand, Rev. Mod. Phys. 88, 015001 (2016), arXiv:1510.05018 [hep-ph]

[12] D. Nogradi and A. Patella, Int. J. Mod. Phys. A31, 1643003 (2016), arXiv:1607.07638 [hep-lat]

[13] A. Hasenfratz, C. Rebbi, and O. Witzel, (2016), arXiv:1609.01401 [hep-ph]

[14] A. Hasenfratz, R. C. Brower, C. Rebbi, E. Weinberg, and O. Witzel, Sakata Memorial KMI Workshop on Origin of Mass and Strong Coupling Gauge Theories (SCGT15) Nagoya, Japan, March 3-6, 2015 (2015), arXiv:1510.04635 [hep-lat]

[15] E. Weinberg, R. C. Brower, A. Hasenfratz, C. Rebbi, and O. Witzel, J. Phys. Conf. Ser. 640, 012055 (2015), arXiv:1412.2148 [hep-lat]

[16] R. C. Brower, A. Hasenfratz, C. Rebbi, E. Weinberg, and O. Witzel, PoS LATTICE2014, 254 (2014), arXiv: 1411.3243 [hep-lat]

[17] R. C. Brower, A. Hasenfratz, C. Rebbi, E. Weinberg, and O. Witzel, J. Exp. Theor. Phys. 120, 423 (2015), arXiv:1410.4091 [hep-lat]

[18] A. Hasenfratz and D. Schaich, (2016), arXiv:1610.10004 [hep-lat]

[19] A. Cheng, A. Hasenfratz, G. Petropoulos, and D. Schaich, JHEP 1307, 061 (2013), arXiv:1301.1355 [hep-lat]

[20] E. Itou, PoS LATTICE2013, 481 (2013), arXiv:1311.2998 [hep-lat]

[21] E. Itou and A. Tomiya, PoS LATTICE2014, 252 (2014), arXiv:1411.1155 [hep-lat]

[22] A. Cheng, A. Hasenfratz, Y. Liu, G. Petropoulos, and D. Schaich, Phys.Rev. D90, 014509 (2014), arXiv:1401.0195 [hep-lat]

[23] M. P. Lombardo, K. Miura, T. J. N. da Silva, and E. Pallante, JHEP 12, 183 (2014), arXiv:1410.0298 [hep-lat]

[24] Z. Fodor, K. Holland, J. Kuti, D. Nogradi, C. Schroeder, K. Holland, J. Kuti, D. Nogradi, and C. Schroeder, Phys. Lett. B703, 348 (2011), arXiv:1104.3124 [hep-lat] 
[25] Z. Fodor, K. Holland, J. Kuti, S. Mondal, D. Nogradi, and C. H. Wong, Phys. Rev. D94, 091501 (2016), arXiv:1607.06121 [hep-lat]

[26] T. DeGrand and A. Hasenfratz, Phys. Rev. D80, 034506 (2009), arXiv:0906.1976 [hep-lat]

[27] L. Del Debbio and R. Zwicky, Phys. Rev. D82, 014502 (2010), arXiv:1005.2371 [hep-ph]

[28] A. Cheng, A. Hasenfratz, G. Petropoulos, and D. Schaich, PoS LATTICE2013, 088 (2013), arXiv:1311.1287 [hep-lat]

[29] A. Hasenfratz, R. Hoffmann, and S. Schaefer, JHEP 0705, 029 (2007), arXiv:hep-lat/0702028 [hep-lat]

[30] J. Osborn, PoS LATTICE2014, 028 (2014)

[31] J. Osborn et al., "Framework for unified evolution of lattices (FUEL),"

[32] R. Narayanan and H. Neuberger, JHEP 0603, 064 (2006), arXiv:hep-th/0601210 [hep-th]

[33] M. Lüscher, Commun.Math.Phys. 293, 899 (2010), arXiv:0907.5491 [hep-lat]

[34] M. Lüscher, JHEP 1008, 071 (2010), arXiv:1006.4518 [hep-lat]

[35] Y. Aoki, T. Aoyama, M. Kurachi, T. Maskawa, K.-i. Nagai, H. Ohki, A. Shibata, K. Yamawaki, and T. Yamazaki (LatKMI), Phys. Rev. D86, 054506 (2012), arXiv:1207.3060 [hep-lat]

[36] Y. Aoki, T. Aoyama, M. Kurachi, T. Maskawa, K.-i. Nagai, H. Ohki, E. Rinaldi, A. Shibata, K. Yamawaki, and T. Yamazaki (LatKMI), Phys. Rev. Lett. 111, 162001 (2013), arXiv:1305.6006 [hep-lat]

[37] K. A. Olive et al. (Particle Data Group), Chin. Phys. C38, 090001 (2014)

[38] R. J. Dowdall, C. T. H. Davies, G. P. Lepage, and C. McNeile, Phys. Rev. D88, 074504 (2013), arXiv:1303.1670 [hep-lat]

[39] G. Ferretti and D. Karateev, JHEP 03, 077 (2014), arXiv:1312.5330 [hep-ph]

[40] L. Vecchi, (2015), arXiv:1506.00623 [hep-ph]

[41] T. Ma and G. Cacciapaglia, JHEP 03, 211 (2016), arXiv:1508.07014 [hep-ph] 